\title{
Scientific Impact of the Operating Temperature for NFIRAOS on TMT
}

\author{
David R. Andersen ${ }^{1, a}$, Glen Herriot ${ }^{1}$, Brent Ellerbroek ${ }^{2}$, and Paul Hickson ${ }^{3}$ \\ 1 NRC Herzberg Institute of Astrophysics, 5071 W. Saanich Rd., Victoria, B.C., V9E 2E7 CANADA \\ 2 TMT Observatory, 2632 E. Washington Blvd, Pasadena, CA 91107, USA \\ 3 Dept. of Physics and Astronomy, 6224 Agricultural Rd., UBC, Vancouver, B.C., V6T 1Z1, CANADA
}

\begin{abstract}
We present the scientific gain enjoyed by client instruments when NFIRAOS is cooled. If NFIRAOS is cooled to $-24^{\circ} \mathrm{C}$, the background contribution of NFIRAOS will not exceed $15 \%$ of the background from sky plus TMT (for a telescope build to current requirements on Mauna Kea). If cooled further to $-30^{\circ} \mathrm{C}$, the K-band inter-OH background-limited exposure times could decrease by more than $7 \%$. We evaluate these gains achieved by cooling NFIRAOS in light of increased technical risks and costs.
\end{abstract}

\section{Introduction}

The first-light adaptive optics facility for TMT will be NFIRAOS[1]. The NFIRAOS design team is guided in our work by a set of top-level TMT requirement documents. These top level requirements ultimately flow down from the TMT science case[2]. The intention of the science case and the toplevel requirements are that subsystems of TMT will all be designed with an eye on maximizing the scientific impact of TMT. An example of such a requirement that forms the basis for this paper is that NFIRAOS shall not increase the (inter-OH) background by more than $15 \%$ of the natural sky plus telescope background for median night-time temperatures on the TMT site. This requirement therefore implies that NFIRAOS be cooled.

There are good technical and scientific reasons to cool NFIRAOS. An isothermal instrument is highly desirable as it makes possible the meeting of tight optical tolerances; if NFIRAOS were warmer than $-10^{\circ} \mathrm{C}$, it would have to have heaters in addition to coolers to keep it isothermal. The TMT Detailed Science Case[2] outlines several examples of TMT science to be done in the inter-OH regions of the K-band:

- metal-free star formation in the early Universe

- spectral dissection of forming galaxies

- extragalactic super-massive black holes

- chemical evolution in nearby galaxies

- initial mass function measured in young clusters.

All of these science cases benefit from having a lower background from NFIRAOS. In total, it is expected that NFIRAOS-fed instruments will be performing K-band spectroscopy $\sim 10 \%$ of the time. Any substantial gains in required exposure time will translate into a more efficient observatory and large savings in operating costs per science exposure.

As pointed out by the NFIRAOS Preliminary Design Review (PDR) panel, however, this requirement to cool NFIRAOS also comes with risks:

- availability of temperature-qualified components

- increased services required

- longer cooling cycling times

- humidity metering and condensation risks on sensitive components.

In this paper, we translate the top-level requirement on the NIR background contribution of NFIRAOS into a proposed operating temperature and assess the scientific impact that this operating temperature will have on the NIR background and required observing times.

a e-mail: david.andersen@nrc-cnrc.gc.ca 


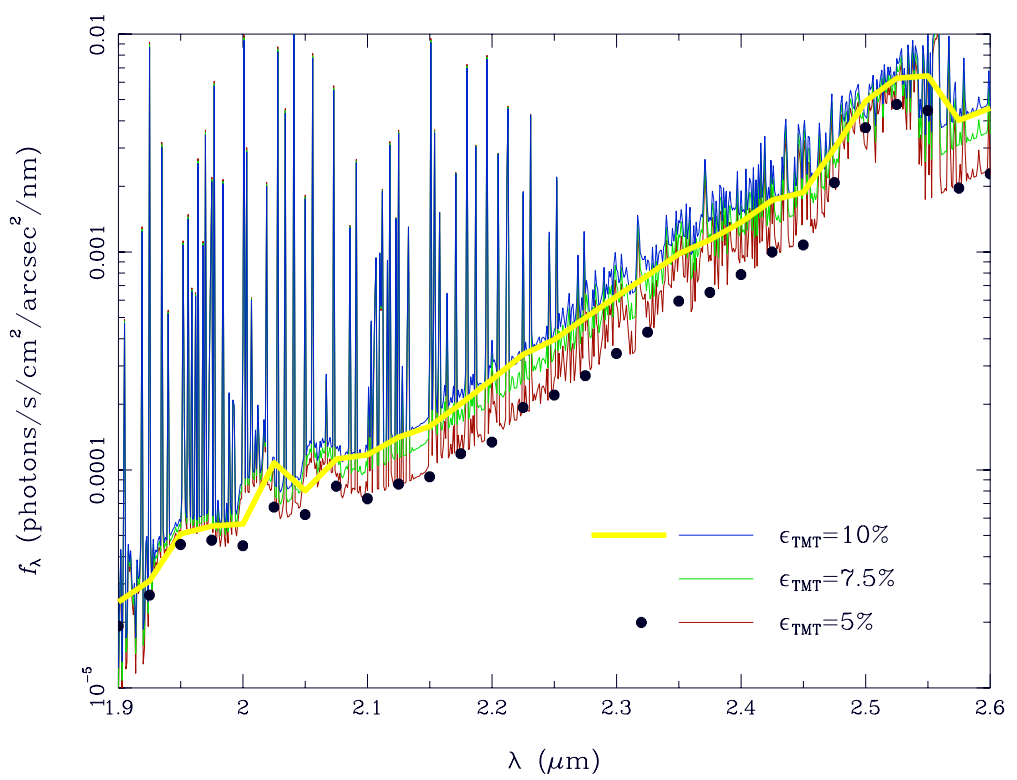

Fig. 1. Sky plus telescope background spectra for $\mathrm{T}_{T M T}=0^{\circ} \mathrm{C}$ and 3 different emissivities listed in the legend. To better quantify the gains in exposure times associated with different NFIRAOS operating temperatures, we sampled the high resolution spectra in 29 wavelength bins between 1.9 and $2.6 \mu \mathrm{m}$ using an iterative clipping algorithm. This binned inter-OH spectra (yellow line for $\epsilon_{T M T}=10 \%$; black dots for $\epsilon_{T M T}=5 \%$ ) is also shown in the figure for comparison.

\section{Sky and Telescope Background}

Our analysis of the scientific impact of the NFIRAOS operating temperature begins at the top of the atmosphere. Using Gemini atmospheric emission and extinction profiles and the requirements and goals for TMT emissivity (TMT is required to have an emissivity of 9\%), we model the sky and telescope background for a variety of night-time temperatures and PWV values.

We use models of the atmospheric emission and extinction that were produced by the Gemini Observatory for use in their Integration Time Calculator (ITC) and are, for the most part, available on their web pages: http:/www.gemini.edu/sciops/telescopes-and-sites/observing-condition-constraints/

Before settling on Mauna Kea (13N) as its site, TMT was also considering Cerro Armazones as a site. Gemini naturally already has models for Mauna Kea, and for the purpose of this exercise we assume that the emission and extinction models of Armazones and Cerro Pachon would be similar.

Mauna Kea has a mean nighttime temperature of $2.3^{\circ} \mathrm{C}$ and a mean Precipital Water Vapor (PWV) of $1.9 \mathrm{~mm}$ while Cerro Armazones has a mean temperature of $7.5^{\circ} \mathrm{C}$ and a mean $\mathrm{PWV}=2.9 \mathrm{~mm}$ [3]. We chose the available Gemini atmospheric emission and extinction models that best meet these expected conditions.

We created high-resolution spectra of the sky plus telescope background (Figure 1) by taking the element-by-element product of the emission and extinction spectra and multiplying by the TMT 
throughput $\left(\tau_{T M T}=1-\epsilon_{T M T}\right)$ and then adding a grey body curve with an appropriate temperature and emissivity:

$$
\begin{gathered}
f_{\text {sky }+T M T}(\lambda)=\tau_{T M T} \tau_{a t m}(\lambda) f_{s k y}(\lambda)+f_{T M T}(\lambda) \\
f_{T M T}(\lambda)=\epsilon_{T M T} 1.41 \times 10^{9} / \lambda^{4} \exp \left[-14400 /\left(273+T_{T M T}\right) / \lambda\right]
\end{gathered}
$$

where all the flux densities mentioned in this document have units photons $/ \mathrm{s} / \operatorname{arcsec}^{2} / \mathrm{cm}^{2} / \mathrm{nm}$, wavelengths are measured in microns and temperatures are measured in degrees Celsius.

As already mentioned, TMT is required to have a throughput of $91 \%$, but we also consider a more ideal telescope with a throughput of $95 \%{ }^{1}$.

To quantify the inter-OH continuum, we again divided the spectra into $49 \times 25 \mathrm{~nm}$ (spanning 1.4 $2.6 \mu \mathrm{m}$ ) subregions. In each subregion, we calculated the iterative mean (using a $2 \sigma$ rejection) while rejecting no values less than the mean. While not perfect, this method for measuring the inter-OH continuum level does provide a good match to the continuum. The only differences in sky plus telescope background as a function of TMT emissivity (or NFIRAOS temperature) occur at wavelengths longer than $1.9 \mu \mathrm{m}$. For most of the analysis that follows, we only consider changes in K-band performance at wavelengths longer than $1.9 \mu \mathrm{m}$.

While the operating temperature of NFIRAOS certainly could have a significant impact on the required exposure times, other factors outside of NFIRAOS will have even greater impact. Given the large number of model sky emission spectra made available to us by Gemini (Tom Geballe, private communication), we make several slices through parameter space to see the impact on exposure time from telescope emissivity, temperature, PWV and airmass (AM). Our reference case for this exploration takes the Mauna Kea site with an $\mathrm{AM}=1, \mathrm{PWV}=1.6 \mathrm{~mm}, \mathrm{~T}_{T M T}=0^{\circ} \mathrm{C}, \epsilon_{T M T}=5 \%$, and a NFIRAOS which strictly meets the requirement that the background due to NFIRAOS not exceed $15 \%$ of the sky plus telescope background in each wavelength bin (once we calculated the NFIRAOS background for this reference case, we used the same background level for all subsequent calculations in this section). We quantified the impact of different observing conditions on the scientific performance for the case of inter-OH background-limited spectroscopy. For this case, the $\mathrm{S} / \mathrm{N}$ in each inter-OH spectral region is defined as:

$$
S / N=f_{\text {source }} \tau_{\text {atm }}(\lambda) \tau_{T M T} \tau_{N F I R A O S} \sqrt{t} / \sqrt{f_{\text {back }}}
$$

where

$$
f_{\text {back }}=\tau_{\text {atm }}(\lambda) \tau_{T M T} \tau_{\text {NFIRAOS }} f_{\text {sky }}+\tau_{\text {NFIRAOS }} f_{T M T}+f_{\text {NFIRAOS }} .
$$

Using this expression, we calculated the ratio of observing time under a given set of observing conditions (varying temperature, AM and PWV for three telescope emissivities) to the reference case, $t / t_{0}$ (Figure 2). The biggest variation, not surprisingly, comes from changing telescope emissivity. Not only is the background increased, but the throughput also falls. A 5\% increase in emissivity corresponds to a $\sim 60 \%$ increase in exposure time. Naturally this difference is even worse when the telescope temperature is greater than $0^{\circ} \mathrm{C}$. From the TMT site testing campaign, we know that the mean nighttime temperature at Mauna Kea will be between roughly $-1^{\circ} \mathrm{C}$ to $5^{\circ} \mathrm{C}$ most of the time [3], and K-band exposure times will need to be $\sim 35 \%$ greater at the warmest temperatures (even higher if the telescope emissivity is not 5\%). Not surprisingly, the other factors we considered, AM and PWV were not as important as temperature and telescope emissivity. Exposure times only varied 10 to $15 \%$ across the expected ranges for these parameters.

\section{The Background Contribution of NFIRAOS}

The background from NFIRAOS is a grey body with $\epsilon=15 \%$ (and $85 \%$ throughput). NFIRAOS is required to contribute less than $15 \%$ of the sky plus TMT background at any wavelength less than

1 There are $3.5 \mathrm{~m}^{2}$ of gaps between TMT primary mirror segments, which will add an additional $0.5 \%$ emissivity (Eric Williams, private communication). We assume the edge of the mirror and the secondary support structure will be properly masked and not contribute further to the thermal emissivity. This small effect is not included in these calculations. 

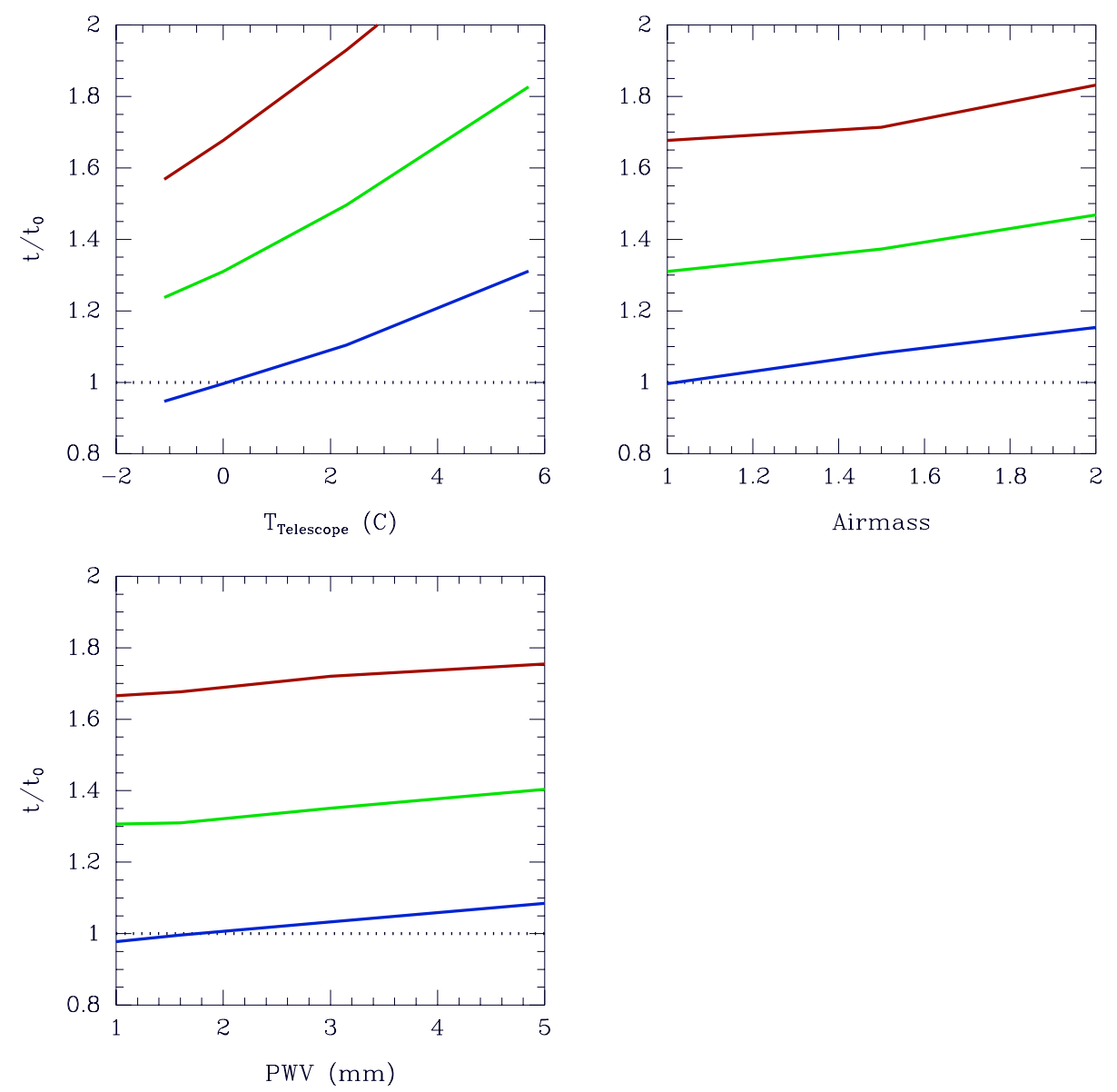

Fig. 2. Variation in relative K-band exposure times versus telescope temperature (top left), airmass (top right) and PWV (bottom left) for three telescope emissivities: 5\% (blue), $7.5 \%$ (green) and 10\% (red). Emissivity is the biggest factor in determining relative exposure times, while temperature also has a big effect. Airmass and PWV have a lesser effect on the required exposure time for background-limited spectroscopy in the inter- $\mathrm{OH}$ regions of the K-band.

$2.5 \mu \mathrm{m}$. Depending on assumptions on the TMT site and emissivity, the NFIRAOS operating temperature needs to be between $-20^{\circ} \mathrm{C}$ and $-30^{\circ} \mathrm{C}$.

The thermal background of NFIRAOS for different temperatures, relative to $15 \%$ of the sky plus telescope background are shown in Figure 3 for TMT on Mauna Kea $\left(0^{\circ} \mathrm{C}\right.$ and $\left.2.5^{\circ} \mathrm{C}\right)$. In the upper left panel of Figure 3, we see that a NFIRAOS operating temperature of $-30^{\circ} \mathrm{C}$ is roughly equal to $15 \%$ of the sky plus telescope background for a telescope with $5 \%$ emissivity. Also notice that different assumptions about the telescope emissivity or site correspond to significantly different NFIRAOS operating temperatures; if the emissivity were higher or TMT is built on Armazones, NFIRAOS could operate at $-20^{\circ} \mathrm{C}$ and still only contribute $\sim 15 \%$ the sky plus telescope background.

To quantify the impact on of the NFIRAOS operating temperature on exposure time and find the temperature which satisfies the requirement that NFIRAOS contribute no more than $15 \%$ of the sky plus telescope background (for different assumptions on the telescope), we again use equation 3 to help 

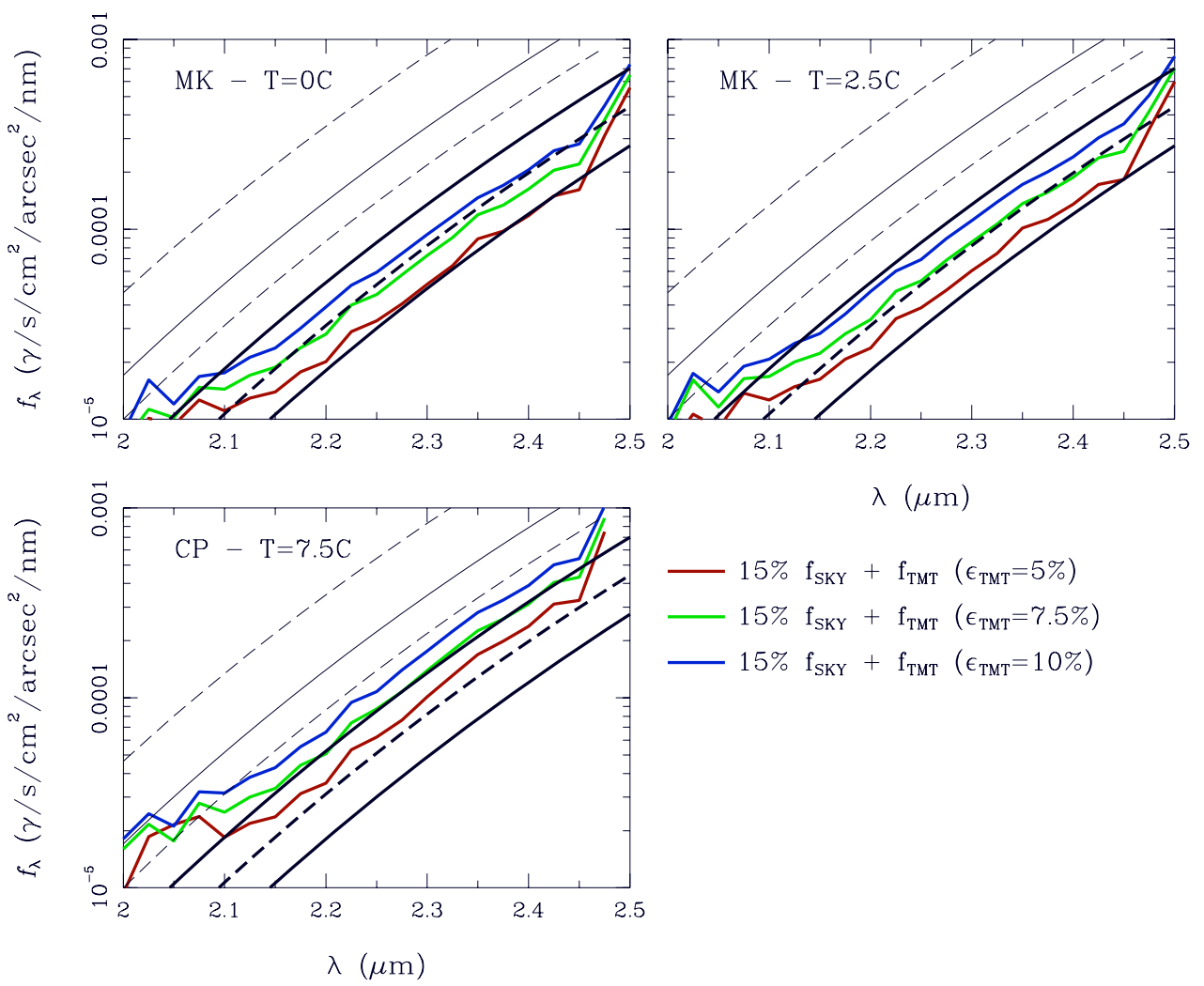

$\lambda(\mu \mathrm{m})$

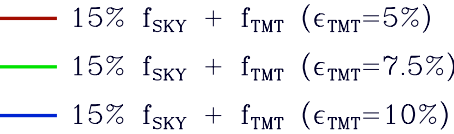

Fig. 3. Grey body flux density curves for NFIRAOS with operating temperatures of $-30^{\circ},-25^{\circ},-20^{\circ},-15^{\circ},-10^{\circ}$, and $0^{\circ} \mathrm{C}$ (solid and dashed lines). Colored curves show $15 \%$ of the sky plus TMT background at Mauna Kea with $\mathrm{T}=0^{\circ} \mathrm{C}$ (upper left panel), $2.5^{\circ} \mathrm{C}$ (upper right panel), and Cerro Pachon with $\mathrm{T}=7.5^{\circ} \mathrm{C}$ (lower left panel).

us define the ratio of exposure times $t / t_{0}$. Again we assume that NFIRAOS contributes a background which is equal to $15 \%$ of the sky plus background flux in each spectral bin for the reference case associated with $t_{0}$.

\section{Results}

Based on three sets of atmospheric conditions and two TMT emissivities, we have arrived at 5 cases to consider. For each of these cases, we determined the temperature for which the maximum $t / t_{0}$ and the weighted mean of $t / t_{0}$ are equal to one (Table 1$)$. In this table,

$$
t / t_{0}(\lambda)=0.85+f_{\text {NFIRAOS }}(\lambda) /\left[\tau_{\text {atm }}(\lambda) \tau_{T M T} f_{\text {sky }}+f_{T M T}(\lambda)\right]
$$

because we are assuming that NFIRAOS contributes $15 \%$ of the sky plus telescope background at every wavelength for the reference case and $\tau_{\text {NFIRAOS }}=85 \%$. Equation 5 also works because we are 
First conference on Adaptive Optics for Extremely Large Telescopes

Table 1. Please write your table caption here.

\begin{tabular}{|c|c|c|c|c|c|c|}
\hline \multirow[b]{2}{*}{ Site } & \multirow{2}{*}{$\begin{array}{l}\text { Telescope } \\
\text { Emissivity }\end{array}$} & \multirow[b]{2}{*}{ Temp $\left({ }^{\circ} \mathrm{C}\right)$} & \multicolumn{2}{|c|}{ NFIRAOS } & \multirow{2}{*}{$\begin{array}{l}\text { Relative } \\
\text { Exposure Time }\end{array}$} & \multirow{2}{*}{$\begin{array}{r}f_{\text {NFIRAOS }} / f_{S K Y}+f_{T M T} \\
\text { does not exceed: }\end{array}$} \\
\hline & & & Emissivity & $\operatorname{Temp}\left({ }^{\circ} \mathrm{C}\right)$ & & \\
\hline Mauna Kea & $5 \%$ & 0 & $15 \%$ & -30 & 1.0 & $17 \%$ \\
\hline Mauna Kea & $9 \%$ & 2.3 & $15 \%$ & -30 & 1.8 & $8 \%$ \\
\hline Mauna Kea & $9 \%$ & 2.3 & $15 \%$ & -24 & 2.0 & $15 \%$ \\
\hline Armazones & $9 \%$ & 7.5 & $15 \%$ & -30 & 3.2 & $5 \%$ \\
\hline Armazones & $9 \%$ & 7.5 & $15 \%$ & -20 & 3.5 & $15 \%$ \\
\hline
\end{tabular}

comparing the change in exposure time for different assumptions about the telescope temperature and emissivity (i.e., the throughputs/emissivities of the telescope and atmosphere in equation 3 are the same for the reference and the measurement and therefore cancel).

We note that we are using the maximum ratio of exposure times in the K-band as the basis for our suggestion of NFIRAOS operating temperature. If we adopt a NFIRAOS operating temperature based on this value, we are saying that NFIRAOS will not contribute more than $15 \%$ of the sky plus telescope background at any wavelength. From 0.8-1.8 $\mu \mathrm{m}$, NFIRAOS will effectively not contribute to the background. In the $\mathrm{K}$-band, from 1.9-2.5 $\mu \mathrm{m}$, the average inter-OH ratio of exposure times would be $4 \%$ less.

Despite this, and despite the fact that NFIRAOS cooled to $-30^{\circ}$ exceeds the requirement on background at all wavelengths for a telescope with $9 \%$ emissivity, there is a roughly $7 \%$ increase in K-band spectroscopic exposure times associated with warming NFIRAOS from $-30^{\circ}$ to $-24^{\circ}$ (Table 1 ). This science penalty combined with the minimal technical risk reduction associated with warming NFIRAOS $6^{\circ}$ have caused us to keep the NFIRAOS operating temperature fixed at $-30^{\circ}$ at this time.

We now summarize the main findings of this study:

- The NFIRAOS PDR review voiced concern about the risks and costs associated with cooling NFIRAOS to $-30^{\circ} \mathrm{C}$. We are working to quantify these technical risks and costs with a special eye on finding any breakpoints with temperature.

- Newly available NIR emission spectra from Gemini suggest the sky background in K-band are quite low. The thermal background from TMT and NFIRAOS will be significant across the entire K-band.

- TMT (not including gaps) has a goal throughput of $94 \%$ and a required throughput of $91 \%$. Gaps should lower this throughput by $0.5 \%$.

- NFIRAOS will have an emissivity of $15 \%$ (throughput of $85 \%$ ).

- Telescope emissivity and temperature have a large impact on the required K-band backgroundlimited exposure times (other factors, including AM and PWV affect the exposure times at a much lower level).

- If the telescope meets the throughput requirement, then NFIRAOS meets its background requirement at a operating temperature of $-24^{\circ} \mathrm{C}$ (Mauna Kea) or $-20^{\circ} \mathrm{C}$ (Cerro Armazones).

- If NFIRAOS were cooled to $-24^{\circ} \mathrm{C}$ to meet the requirement on background assuming median nighttime temperatures on Mauna Kea and a $9 \%$ telescope emissivity, K-band spectroscopic exposure times would have to be $7 \%$ longer than if NFIRAOS were cooled to $-30^{\circ}$.

\section{References}

1. Herriot, G. et al. (2006), Proceeding of the SPIE, 6272, 62720Q

2. TMT Detailed Science Case (2007), www.tmt.org/foundation-docs/TMT-DSC-2007-R1.pdf

3. Schöck, M. et al. (2009), PASP, 121, 384 